\title{
Dissociating Response Conflict and Error Likelihood in Anterior Cingulate Cortex
}

\author{
Nick Yeung ${ }^{1}$ and Sander Nieuwenhuis ${ }^{2}$ \\ ${ }^{1}$ Department of Experimental Psychology, University of Oxford, Oxford OX1 3UD, United Kingdom, and 2Institute of Psychology and Leiden Institute for \\ Brain and Cognition, Leiden University, 2333 AK, Leiden, The Netherlands
}

\begin{abstract}
Neuroimaging studies consistently report activity in anterior cingulate cortex (ACC) in conditions of high cognitive demand, leading to the view that ACC plays a crucial role in the control of cognitive processes. According to one prominent theory, the sensitivity of ACC to task difficulty reflects its role in monitoring for the occurrence of competition, or "conflict," between responses to signal the need for increased cognitive control. However, a contrasting theory proposes that ACC is the recipient rather than source of monitoring signals, and that ACC activity observed in relation to task demand reflects the role of this region in learning about the likelihood of errors. Response conflict and error likelihood are typically confounded, making the theories difficult to distinguish empirically. The present research therefore used detailed computational simulations to derive contrasting predictions regarding ACC activity and error rate as a function of response speed. The simulations demonstrated a clear dissociation between conflict and error likelihood: fast response trials are associated with low conflict but high error likelihood, whereas slow response trials show the opposite pattern. Using the N2 component as an index of ACC activity, an EEG study demonstrated that when conflict and error likelihood are dissociated in this way, ACC activity tracks conflict and is negatively correlated with error likelihood. These findings support the conflict-monitoring theory and suggest that, in speeded decision tasks, ACC activity reflects current task demands rather than the retrospective coding of past performance.
\end{abstract}

\section{Introduction}

Human neuroimaging studies indicate that anterior cingulate cortex (ACC) plays a critical role in the organization of thought and action. In particular, EEG and fMRI studies consistently report increased ACC activity in difficult or demanding conditions: when people try to override habitual actions, when they select between multiple response alternatives, and when they make errors (Botvinick et al., 2001). For example, robust ACC activity is observed in the flanker task when distractor stimuli cue a different (incongruent) response to the central target, relative to the case in which distractors cue the same (congruent) response (Botvinick et al., 1999). In the scalp-recorded EEG, increased cognitive demand is reflected in the N2 component, an enhanced negativity over frontocentral sites. EEG source localization (Van Veen and Carter, 2002; Nieuwenhuis et al., 2003; Yeung et al., 2004), convergence with fMRI findings (Carter et al., 1998), and intracranial studies (Wang et al., 2005) all identify ACC as the source of the N2.

According to one prominent theory, the sensitivity of ACC to task demand reflects its role in monitoring for response conflict, the coactivation of mutually incompatible actions (Carter et al., 1998; Botvinick et al., 2001). This theory explains the N2, for

Received July 27, 2009; revised Sept. 29, 2009; accepted 0ct. 2, 2009

N.Y. was supported by National Institutes of Health Grant P50-MH62196; S.N. was supported by a Netherlands Organisation for Scientific Research NWO-VIDI grant. We thank Nisan Mol for assistance in running the EEG study.

Correspondence should be addressed to Nick Yeung, Department of Experimental Psychology, University of Oxford, South Parks Road, 0xford 0X1 3UD, UK. E-mail: nicholas.yeung@psy.ox.ac.uk.

D01:10.1523/JNEUROSCI.3615-09.2009

Copyright $\odot 2009$ Society for Neuroscience ～0270-6474/09/2914506-05\$15.00/0 example, in terms of conflict between competing responses associated with target and flanker stimuli on incongruent trials (Yeung et al., 2004). Through this conflict-monitoring function, ACC is held to play a key role in the regulation of thought and action by signaling the need for increased attentional control (Kerns et al., 2004).

However, another broad class of theories offers an alternative account of observed ACC activity, based on the notion that this region is involved in the control of action selection via reinforcement learning (Holroyd and Coles, 2002; Brown and Braver, 2005). Specifically, ACC has been proposed to use reward-related dopamine signals to generate predictions of error likelihood (Brown and Braver, 2005). According to this theory, ACC activity observed in difficult task conditions-such as incongruent trials in the flanker task - reflects a prediction of high error likelihood, rather than the signaling of response conflict per se. This hypothesis therefore offers an alternative account of apparent conflictrelated activity - as a byproduct of coding error likelihood-that recasts the functional role of ACC as recipient, rather than key source, of performance-monitoring signals.

Although the conflict and error-likelihood theories are not necessarily mutually exclusive, and it is unlikely that ACC performs a unitary function, the two theories nevertheless offer sharply contrasting accounts of ACC sensitivity to task difficulty, a canonical feature of ACC function. However, despite clear conceptual differences, the theories are difficult to distinguish empirically because conflict and error likelihood strongly correlate: both typically increase with increasing cognitive demand. In the present study we therefore combined detailed computational 
a

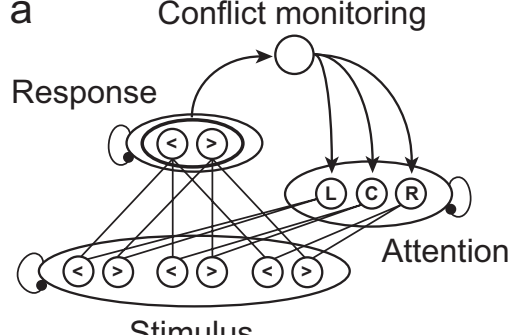

b

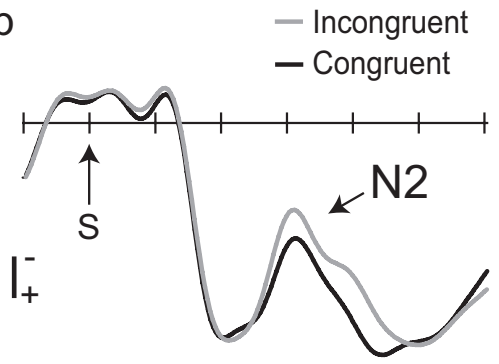

Figure 1. Conflict and the N2. $\boldsymbol{a}$, Schematic model of response selection and conflict monitoring in the flanker task. $\boldsymbol{b}$, Stimulus-locked potentials in the flanker task, recorded at frontocentral electrode FCz. Intervals of $100 \mathrm{~ms}$ are marked on the abscissa.

simulations and EEG methods to identify and test contrasting predictions of the theories. Our simulations suggest a critical dissociation between conflict and error likelihood as a function of response speed. Our EEG study, focusing on the flanker-related $\mathrm{N} 2$, evaluated the contrasting predictions to test between the competing accounts.

\section{Materials and Methods}

The present study is founded on two straightforward observations about human performance. First, accuracy typically improves when people take longer to make a given decision (i.e., as response time, $R T$, increases). Thus, within experimental conditions, fast responses are associated with more errors than are slow responses-the ubiquitous speed-accuracy trade-off. Second, RT typically increases with the degree of conflict or uncertainty about the correct response-fast responses are associated with less conflict than are slow responses. Together, these two features of human performance suggest a critical dissociation between conflict and error likelihood within experimental conditions: On trials with fast responses, conflict should be low whereas error likelihood should be high. In contrast, on trials with slow responses, conflict should be high whereas error likelihood should be low. Therefore, whereas conflict and error likelihood are typically confounded across conditions (e.g., comparing congruent and incongruent trials), they should dissociate as a function of $\mathrm{RT}$ within individual conditions. In what follows, we describe our efforts to formalize these predictions using computational modeling and to evaluate the predictions in an empirical EEG study. Additional details of the simulation and EEG methods and results are provided as supplemental material, available at www.jneurosci.org.

\section{Simulation methods}

Our simulations made use of an established connectionist model of flanker-task performance, in which stimulus information feeds into a competitive process of response selection under the influence of attention (Fig. 1a). This model has previously been shown to accurately simulate a range of behavioral and EEG findings from the flanker task (Spencer and Coles, 1999; Botvinick et al., 2001). Our own previous work with this model has focused on simulating ACC function according to the conflict-monitoring theory (Yeung et al., 2004; Yeung and Cohen, 2006). The present simulations aimed, first, to implement a corresponding version of the error-likelihood theory and, second, to contrast the predictions of this model with those of the conflict-monitoring theory.
Model architecture

Task network. Three layers of units-stimulus, response, and attention layers_-implement task performance. Stimuli are represented as patterns of activity across an input layer containing units corresponding to each stimulus type (here, letters and numbers) in each of three locations. Activity from this layer flows through connections to the response layer, which has units corresponding to left- and right-hand responses. Input from the attention layer boosts activation of central target stimuli relative to the distracting flankers, ensuring that the model usually produces the correct response even on incongruent trials.

Conflict monitoring. As in previous simulations (Botvinick et al., 2001; Yeung et al., 2004), conflict was formalized as the product of the activation levels of competing response units, scaled by the strength of the inhibitory connection between them:

$$
\text { Conflict }=-\sum_{i=1}^{N} \sum_{j=1}^{N} a_{i}{ }^{\star} a_{j}{ }^{*} w_{i j},
$$

where $a$ denotes unit activity, $w$ the connection weight between units, and the subscripts $i$ and $j$ are indexed over the units of interest (here, the two response units). This product measure captures the concept of response conflict in a simple way: On incongruent trials, target and flankers activate different responses, the product of response activation levels is therefore large, and conflict is high. On congruent trials, target and flankers activate the same (correct) response, incorrect response activity is low or zero, the product of activation levels is low or zero, and there is little or no conflict. Simulated response conflict is entirely governed by parameters of the task network; no additional free parameters are used to implement the conflict-monitoring system.

\section{Error likelihood}

The error-likelihood theory has previously been implemented using a complex nonlinear dynamical system model, in which ACC activity is simulated across a population of rate-coded units (Brown and Braver, 2005). The theory has not been implemented for the flanker task, and the original model is substantially more complex than the corresponding conflict model. We therefore implemented an abstracted model that captures in a simple and transparent way the central claim of the errorlikelihood theory, that ACC activity reflects the previous probability of errors in particular stimulus contexts. Thus, ACC activity was simulated as reflecting an exponentially weighted history of error likelihood for each of the four stimulus types (congruent and incongruent trials with letter and number target stimuli) according to the following equation:

$$
E L_{S, n}=\lambda * E r r_{n}+(1-\lambda) \star E L_{S, n-1},
$$

where $E L$ denotes perceived error likelihood for a given stimulus type, $S$, after the current trial $n . \lambda$ is a learning rate parameter, and Err takes a value of 1 or 0 for error and correct responses, respectively. Thus, errorlikelihood estimates for the presented stimulus type were increased after each error and decreased after each correct response such that, with learning, the estimate approaches the objective error rate. $\lambda$ is the only free parameter in this part of the model. Simulation results were essentially identical across variations in $\lambda$, reflecting the stable task environment being simulated. In the reported simulations, $\lambda$ took a value of 0.5 .

\section{EEG methods}

Participants. The 16 participants in the EEG study, 10 women and 6 men, ages $18-31$ years $($ mean $=24.6)$, were paid for their involvement and gave informed consent.

Procedure. Participants performed a version of the flanker task in which they responded with a left or right button press to indicate whether the central item in a 3-character stimulus string was a letter or a number. The letters used were C, G, H, L, N, Q, P, and R, and the numbers were the digits from 2 to 9 . The stimulus on each trial contained 3 different characters, a central target and two flankers. The target was either a letter or a number; the flankers were either two letters or two numbers. Half of the stimuli were congruent (comprising all numbers or all letters) and half were incongruent (comprising a letter flanked by numbers or a number flanked by letters). Letter and number stimuli were selected randomly on 
Table 1. Mean RTs and error rates in the empirical data (upper rows) and simulated data (lower rows)

\begin{tabular}{lccr}
\hline & RT (cycles) & RT (ms) & Error (\%) \\
\hline Empirical & & & \\
$\quad$ Congruent & - & $427(12)$ & $8.1(0.7)$ \\
$\quad$ Incongruent & - & $452(13)$ & $15.8(0.9)$ \\
Simulation & & & \\
$\quad$ Congruent & $9.7(0.22)$ & $427(5)$ & $7.9(0.8)$ \\
$\quad$ Incongruent & $10.8(0.22)$ & $452(5)$ & $16.6(1.1)$ \\
\hline
\end{tabular}

SEs are given in parentheses. - , No RT (cycle) value for the empirical data.

each trial, with the constraint that the three characters presented on trial $\mathrm{N}$ could not appear on trial $\mathrm{N}+1$.

On each trial participants were first presented with a fixation marker " **** for $500 \mathrm{~ms}$. The stimulus then appeared in the center of the screen for $100 \mathrm{~ms}$, at which point the screen cleared until 500-700 ms after the participant's response, followed by the fixation marker for the subsequent trial. Participants were first given 40 trials of practice, repeated if necessary. They then performed 16 blocks of 104 trials. For half of the blocks, $80 \%$ of trials were congruent and $20 \%$ were incongruent, and for the other half of the blocks these proportions were reversed. This frequency manipulation was not central to the present concerns, so the analysis below collapses across frequency conditions. Analyses (and corresponding simulations) including only frequent trial conditions yielded comparable results.

\section{EEG recording and analysis}

EEG data were collected with SynAmps amplifiers (Neuroscan), from 30 scalp locations, using $\mathrm{Ag} / \mathrm{AgCl}$ electrodes embedded in a fabric cap. The data were off-line low-pass filtered $<12 \mathrm{~Hz}$. For correct trials only, stimulus-locked epochs of $1500 \mathrm{~ms}(-500$ to $+1000 \mathrm{~ms}$; baseline of -100 to $0 \mathrm{~ms}$ ) were extracted from the continuous EEG data for further analysis. The N2 was evident in the grand-averaged event-related potential (ERP) waveform as a negative-going deflection, peaking $\sim 300 \mathrm{~ms}$ poststimulus, that was enhanced on incongruent trials relative to congruent trials (Fig. 1b). We quantified the N2 as the base-to-peak voltage difference between the most negative peak in the window from 150 to 500 $\mathrm{ms}$ poststimulus and the immediately preceding positive peak. Degrees of freedom were corrected using Greenhouse-Geisser $\varepsilon$ values where appropriate.

\section{Results}

\section{Behavioral data and model fit}

RTs and error rates in the empirical and simulated datasets are given in Table 1. Analysis of the empirical data revealed reliable effects of congruence on RTs, $t_{(15)}=7.76, p<0.01$, and error rates, $t_{(15)}=8.11, p<0.01$, indicating poorer performance on incongruent than congruent trials. As in previous research (Yeung et al., 2004; Yeung and Cohen, 2006), we fit the model to the behavioral data, in particular the observed error rates, as a basis for assessing model predictions regarding the EEG data. Table 1 indicates that the model captured the main features of behavioral performance, with simulation values falling within a SE of corresponding values for the empirical RTs and error rates. Given this satisfactory fit to the behavioral results, of interest are the model predictions regarding the N2.

\section{Simulation results}

To analyze the relationship between conflict, error likelihood and response speed and accuracy, simulated trials were sorted into quintile bins according to RT, separately for congruent and incongruent trials. Response accuracy, response conflict, and perceived error likelihood were then calculated for each bin, with conflict and error likelihood estimates measured for correct trials only (paralleling our analysis of the empirical N2 below). The

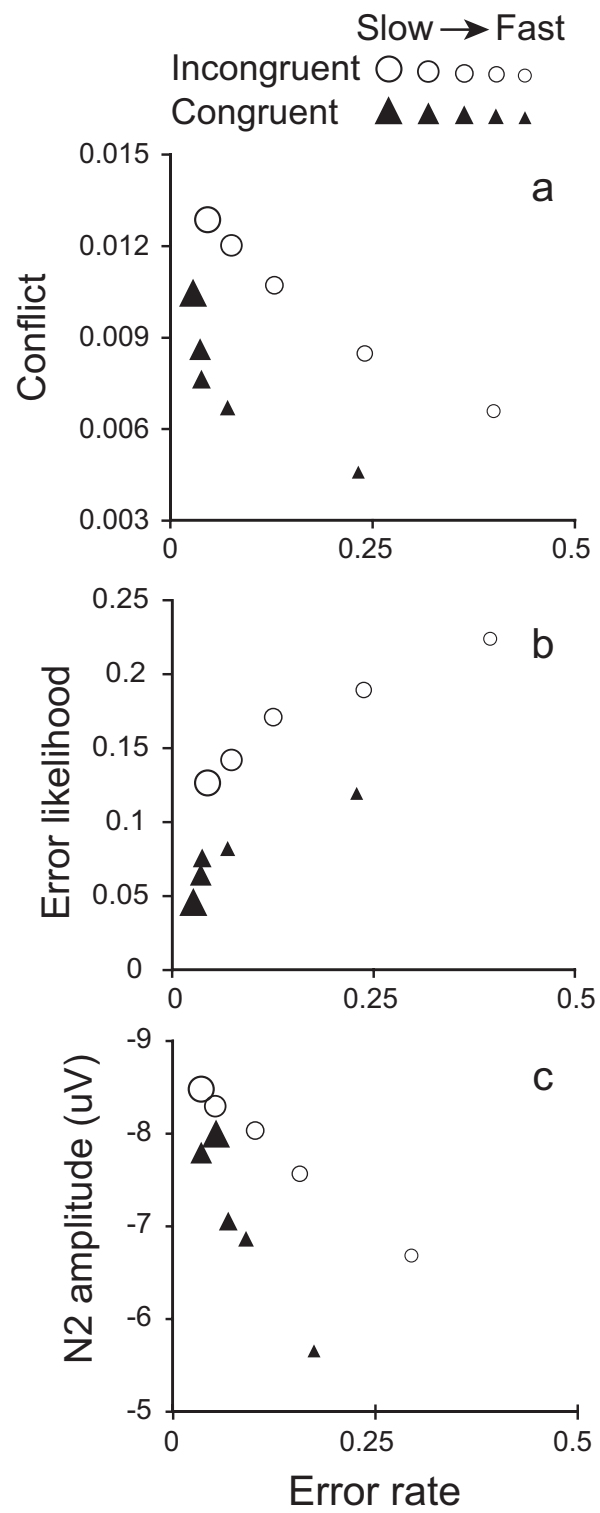

Figure 2. Predicted and observed ACC activity as a function of error proportion. Data are plotted for simulated conflict $(\boldsymbol{a})$, simulated error likelihood (b), and observed $\mathrm{N} 2$ amplitude (c), for correct response trials in each of five RT quintile bins.

results (Fig. 2) confirm that the conflict and error-likelihood theories both predict increased ACC activity on incongruent relative to congruent trials, but that they make very different predictions regarding variability in ACC activity as a function of RT within each condition.

In the conflict-monitoring simulation, trials with the lowest proportion of errors-the slowest trials-were associated with the highest levels of conflict (Fig. 2a). As error rate increased-for faster RT bins-levels of conflict decreased, for both congruent and incongruent stimuli. As described above, these results follow from two fundamental features of human performance that are replicated in our computational model: Speed and accuracy trade off such that slower responses tend to be more accurate, and RTs increase with the degree of conflict experienced. Thus, the conflict-monitoring theory predicts that, within experimental conditions, ACC activity should vary inversely with error rate.

In contrast, and as one would intuitively predict, the errorlikelihood theory predicts the opposite pattern, with perceived 
error likelihood increasing as a function of actual error rate (Fig. $2 b$ ). Although predicted, it is notable that this pattern emerges even though our model generates estimates of error likelihood for each stimulus type regardless of trial RT (cf. Eq. 2). This pattern is not driven by changes in estimated error likelihood on error trials themselves, since error trials were excluded from the analysis. Instead, the critical factor is that model performance is broadly stable over time, such that fast responses tend to be preceded by other fast responses. Critically, these other fast responses also have a higher probability of being incorrect-reflecting the ubiquitous speed-accuracy trade-off. It follows that, by the time the model produces a fast response to a particular stimulus, the error likelihood associated with this stimulus (determined on the basis of recent performance) tends to be increased. Thus, estimates of error likelihood vary within stimulus conditions because fast responses occur more frequently during periods of high error likelihood than slow responses. To the degree that ACC tracks error likelihood, activity in this region should therefore increase for fast responses (where errors are likely) relative to slow responses (where errors are infrequent).

Thus, the conflict and error-likelihood theories make strikingly different predictions about the way ACC activity should vary as a function of accuracy and response speed. These predictions are very robust; for example, they are observed regardless of whether attention levels are stable across trials, as in the simulation data in Figure 2, or vary as a function of experienced conflict or error likelihood (supplemental material, available at www. jneurosci.org). Of interest, then, is the nature of this relationship for the empirical N2.

\section{EEG data}

Grand-averaged ERPs for congruent and incongruent stimuli, for correct trials only, are presented in Figure $1 b$. Replicating previous research, N2 amplitude was greater on incongruent trials than on congruent trials, $F_{(1,15)}=9.93, p<0.01, \eta_{p}^{2}=0.40$, and this $\mathrm{N} 2$ enhancement was greater at frontal sites than posterior sites, $F_{(4,60)}=5.76, p<0.01, \eta_{p}^{2}=0.28, \varepsilon=0.65$.

As described above, this difference in N2 amplitude between congruent and incongruent trials is consistent with both the conflict and error-likelihood theories. However, the theories make crucially different predictions about the way N2 amplitude should vary as a function of error rate and response speed within conditions. To analyze this relationship, the behavioral and EEG data were sorted into quintile bins on the basis of RT, separately for each participant and for congruent and incongruent trials. Stimulus-locked ERP waveforms were calculated for each bin, for correct trials only, yielding 10 waveforms per participant ( 5 quintiles each for congruent and incongruent trials). For each condition, we then calculated the error rate and correct-trial N2 amplitude (at electrode $\mathrm{FCz}$, where the $\mathrm{N} 2$ was most pronounced). Figure $2 c$ plots the resulting values, averaged across participants.

As shown in Figure $2 c$, a strong negative correlation between error rate and N2 amplitude was clearly evident. This negative correlation was reliable for both congruent trials, $r=-0.96, p<$ 0.01 , and incongruent trials, $r=-0.99, p<0.01$. Thus, trials with the lowest error rate (in the slowest RT bin) were associated with the greatest N2 amplitude, whereas trials with the highest error rate (in the fastest RT bin) were associated with the lowest $\mathrm{N} 2$ amplitude, consistent with the predictions of the conflict theory. To assess the robustness of these effects across participants, we performed separate correlation analyses for the error rate and N2 data of each participant. Across participants, the correlation coefficients were consistently negative for both congruent trials (mean $r=-0.47 ; r<0$ for $13 / 16$ participants, sign test $p<0.05$ ) and incongruent trials (mean $r=-0.45 ; r<0$ for $14 / 16$ participants, sign test $p<0.05$ ).

Further analyses are described in the supplemental material, available at www.jneurosci.org. These analyses demonstrate that the observed relationship between N2 amplitude and error rate was not driven by differential overlap with response-related EEG activity, that N2 amplitude was insensitive to trial-to-trial variability in error likelihood, and that the conflict-monitoring theory is able to account for RT-related variability in a second EEG index of ACC activity, the error-related negativity (although here the conflict and error-likelihood theories do not make differential predictions).

\section{Discussion}

The conflict and error-likelihood theories provide contrasting accounts of perhaps the most replicated neuroimaging finding regarding ACC function: its sensitivity to task difficulty. According to the conflict theory, this sensitivity reflects the role of ACC in monitoring for conflict between competing responses when decision making is difficult or uncertain. According to the errorlikelihood theory, ACC activity reflects prior learning about the frequency of errors in these situations. Conflict and error likelihood are confounded in most experimental contrasts because errors are likely in conditions of response conflict. However, our computational simulations demonstrate that conflict and error likelihood dissociate as a function of response speed. Our empirical EEG data demonstrate that when response conflict and error likelihood are dissociated in this way, ACC activity tracks the level of conflict, not error likelihood.

The conflict-monitoring theory predicts that ACC activity should be greatest on trials with the longest RTs-which are the trials with the lowest error rates - and should be reduced on faster, less accurate trials. It may seem somewhat counterintuitive that trials with the highest conflict should produce the fewest errors. In our simulations, this feature follows from the fact that activity in the target stimulus unit and correct response unit tend to increase over time, as stimulus processing progresses under the influence of attention. As a consequence, although trials with long RTs tend to have high levels of conflict, the responses ultimately made tend to be correct. In contrast, very fast RTs occur when processing noise causes "fast guess" responses. Fast guesses tend to be inaccurate, by definition, and are associated with little conflict because responses occur before significant conflict develops. Thus, conflict is negatively correlated with error rate.

In contrast, the error-likelihood theory predicts that ACC activity should increase with error rate. In our simulations, the model develops accurate estimates of error likelihood to the extent that there is stability in performance accuracy over time, such that accuracy on the current trial is correlated with past performance. Such stability is introduced, for example, through speed-accuracy trade-offs whereby periods of fast responding are associated with increased error rates. Any systematic variability of this kind will reinforce the positive correlation between actual and perceived error rate. Given that systematic variability is a ubiquitous feature of human performance (Gilden, 2001) and is evident in the present data-for example, as a block-wise speed-accuracy tradeoff-the error-likelihood theory should predict a positive correlation between ACC activity and observed error rate.

Conflict and error likelihood therefore vary in opposing ways as a function of response speed. Our empirical data provided clear evidence that ACC activity follows the predictions of the 
conflict-monitoring theory: N2 amplitude was markedly larger on trials with long RTs (for which conflict is high and error likelihood is low) than on trials with short RTs (for which conflict is low and error likelihood is high). These findings suggest strongly that ACC activity in the flanker task reflects the current level of cognitive demand-increasing with the degree of response conflict (Botvinick et al., 2001; Yeung et al., 2004) —rather than retrospectively coding past performance on the basis of gradual reinforcement learning (Brown and Braver, 2005). Inspection of detailed patterns in the EEG data (Fig. $2 c$ ) emphasizes this point. Thus, although errors were consistently more likely on incongruent trials than on congruent trials, N2 amplitude on some congruent trials clearly exceeded $\mathrm{N} 2$ amplitude on some incongruent trials. Indeed, strikingly, the N2 was larger on slow congruent trials - the condition with highest response accuracy-than on fast incongruent trials - the condition with lowest accuracy.

The observed negative correlation between ACC activity and error rate presents a clear challenge to the error-likelihood theory. It nonetheless remains possible that error likelihood is coded in some regions of medial prefrontal cortex, but that this coding is not reflected in the broad, spatially summated activity measured in the scalp-recorded EEG. According to this interpretation, subregions in ACC or neighboring cortex may show sensitivity to error likelihood as well as response conflict, but the former regions might only be visible to methods such as fMRI with finer-grained spatial resolution (Brown and Braver, 2007; Brown, 2009). In the context of findings of multiple subregions of activity within ACC, the present research suggests a simple discriminative test for identifying whether activated regions are sensitive to conflict or error likelihood: As we have demonstrated, conflict-sensitive regions should show increased activity as a function of RT within conditions, whereas regions sensitive to error likelihood should show the opposite pattern.

More broadly, the present research illustrates the value of combining computational and neuroimaging approaches in the development of theories of cognitive function. In the present research, computational simulations played a crucial role in identifying contrasting predictions of the competing theories. Future research using this combined approach might profitably address the question of how we might reconcile the findings of the present study - which suggest that ACC activity in speeded decision tasks reflects current cognitive demands rather than past performance-with an emerging consensus that ACC plays an important role in value-based decision making (e.g., Rushworth et al., 2004). These differing interpretations may at least in part reflect the distinct methodological approaches used to study ACC function in different contexts: speeded decisions in which the correct response is known (as in the present study) versus reward-guided decisions in which the correct response has to be learned. In this regard, we concur with recent suggestions that apparent discrep- ancies regarding ACC function are interpretable within a common underlying framework, in which ACC contributes broadly to the optimization of decision processes in the context of cognitive and environmental demands (Botvinick, 2007). On this view, monitoring for conflict during decision making, as observed in the present study, would represent one valuable source of information used by ACC in this optimization process.

\section{References}

Botvinick M, Nystrom LE, Fissell K, Carter CS, Cohen JD (1999) Conflict monitoring versus selection-for-action in anterior cingulate cortex. Nature 402:179-181.

Botvinick MM (2007) Conflict monitoring and decision making: reconciling two perspectives on anterior cingulate function. Cogn Affect Behav Neurosci 7:356-366.

Botvinick MM, Braver TS, Carter CS, Barch DM, Cohen JD (2001) Conflict monitoring and cognitive control. Psychol Rev 108:624-652.

Brown JW (2009) Conflict effects without conflict in anterior cingulate cortex: multiple response effects and context-specific representations. Neuroimage 47:334-341.

Brown JW, Braver TS (2005) Learned predictions of error likelihood in the anterior cingulate cortex. Science 307:1118-1121.

Brown JW, Braver TS (2007) Risk prediction and aversion by anterior cingulate cortex. Cogn Affect Behav Neurosci 7:266-277.

Carter CS, Braver TS, Barch DM, Botvinick MM, Noll D, Cohen JD (1998) Anterior cingulate cortex, error detection, and the online monitoring of performance. Science 280:747-749.

Gilden DL (2001) Cognitive emissions of 1/f noise. Psychol Rev 108:33-56.

Holroyd CB, Coles MG (2002) The neural basis of human error processing: Reinforcement learning, dopamine, and the error-related negativity. Psychol Rev 109:679-709.

Kerns JG, Cohen JD, MacDonald AW 3rd, Cho RY, Stenger VA, Carter CS (2004) Anterior cingulate conflict monitoring and adjustments in control. Science 303:1023-1026.

Nieuwenhuis S, Yeung N, van den Wildenberg W, Ridderinkhof KR (2003) Electrophysiological correlates of anterior cingulate function in a $\mathrm{Go} /$ NoGo task: effects of response conflict and trial-type frequency. Cogn Affect Behav Neurosci 3:17-26.

Rushworth MF, Walton ME, Kennerley SW, Bannerman DM (2004) Action sets and decisions in the medial frontal cortex. Trends Cogn Sci $8: 410-417$.

Spencer KM, Coles MG (1999) The lateralized readiness potential: Relationship between human data and response activation in a connectionist model. Psychophysiology 36:364-370.

Van Veen V, Carter CS (2002) The timing of action monitoring in rostral and caudal anterior cingulate cortex. J Cogn Neurosci 14:593-602.

Wang C, Ulbert I, Schomer DL, Marinkovic K, Halgren E (2005) Responses of human anterior cingulate cortex microdomains to error detection, conflict monitoring, stimulus-response mapping, familiarity, and orienting. J Neurosci 25:604-613.

Yeung N, Cohen JD (2006) The impact of cognitive deficits on conflict monitoring: predictable dissociations between the error-related negativity and N2. Psychol Sci 17:164-171.

Yeung N, Botvinick MM, Cohen JD (2004) The neural basis of error detection: conflict monitoring and the error-related negativity. Psychol Rev 111:931-959. 